\title{
Application of Dvoretzky's Theorem of Measure Concentration in Physics and Cosmology
}

\author{
Mohamed S. El Naschie \\ Department of Physics, University of Alexandria, Alexandria, Egypt \\ Email: Chaossf@aol.com
}

Received 22 October 2015; accepted 26 October 2015; published 29 October 2015

Copyright (C) 2015 by author and Scientific Research Publishing Inc.

This work is licensed under the Creative Commons Attribution International License (CC BY). http://creativecommons.org/licenses/by/4.0/

\section{(c) (i) Open Access}

\begin{abstract}
Using Dvoretzky's theorem in conjunction with Bohm's picture of a quantum particle inside a guiding quantum wave akin to De Broglie-Bohm pilot wave we derive Einstein's famous formula $E$ $=m c^{2}$ as the sum of two parts $E(O)=m c^{2} / 22$ of the quantum particle and $E(D)=m c^{2}(21 / 22)$ of the quantum wave where $m$ is the mass, $c$ is the speed of light and $E$ is the energy. In addition we look at the problem of black holes information in the presence of extra dimensions where it seems initially that extra dimensions would logically lead to a hyper-surface for a black hole and consequently a reduction of the corresponding information density due to the dilution effect of these additional dimensions. The present paper argues that the counterintuitive opposite of the above is what should be expected. Again this surprising result is a consequence of the same well known theorem on measure concentration due to I. Dvoretzky. We conclude that there are only two real applications of the theorem and we expect that many more applications in physics and cosmology will be found in due course.
\end{abstract}

\section{Keywords}

Spacetime Extra Dimensions, Dvoretzky's Theorem, Information Paradox, E-Infinity Theory, Counterintuitive Geometry, Dark Energy of the Quantum Wave, 'tHooft-Susskind Black Holes, Wave-Particle Duality, De Broglie-Bohm Pilot Wave

\section{Introduction}

The main purpose of the present paper is to show that Dvoretzky's theorem is not only pure mathematics but also has profound applications in physics and cosmology. For instance it would be intuitively reasonable to sup- 
pose that in the case of black holes [1]-[10] with high dimensionality, i.e. extra dimensions [8], the horizon i.e. the hyper quasi surface of the black hole horizon will also be of higher dimensionality. Therefore it would seem to follow that the information that resides only on the surface will be, so to speak, diluted because the Bekenstein limit is supposed to remain the same [1] [3]. In other words the net effect is that the information density will decrease or so it would seem initially [10].

In the present short work however we show that due to the above mentioned well known theorem of Dvoretzky on measure concentration the above conclusion is fallacious [11]-[16]. The said theorem due to the legendary Ukrainian-Israeli mathematician and past time advisor of the Government for armaments and President of the famous Weizmann Institute, I. Dvoretzky [16] [17] leads to the definite conclusion that in sufficiently high dimensional Banach-like spaces such as our quantum spacetime [12]-[14], about 96\% of the volume resides on the surface or very near to it while a near to only 4\% remains in the deceptive bulk [16] [17]. An almost identical result may be obtained using E-infinity theory [11]-[14] with regard to energy where the 96\% energy residing on the surface is identified with the supposedly missing so called dark energy [12] [13]. Consequently using the Bohm picture or more accurately, the De Broglie-Bohm pilot wave theory of a quantum particle surrounded by a guiding quantum wave we come to a profound conclusion using Dvoretzky's theorem. Noting the well know connection between information, entropy and thus thermodynamics and energy [2] [6] [7] we see that our conclusion has an indirect actual cosmic measurement and observational justification, in fact, confirmation [11] [12] [17]. In addition we note parenthetically that the Bekenstein wonderful result [1]-[8] upon which we are basing ourselves still needs an extension to a fractal version [11] by means of which the reduction in the information density will also be excluded [11]-[17]. It is thought that in this form the Bekenstein real limit on information will become topological and measure theoretical universality which dispels the black hole information paradox [6] [7] [11] [12] in an unheard of simplicity conserving the most important feature of the theory of Steven Hawking and Jacob Bekenstein on the one side and 'tHooft-Susskind on the other without violating any fundamental laws of physics [11] [12]. This and more is the consequence of the marvellous theorem of the great mathematician I. Dvoretzky.

\section{Analysis}

We will start in the present work with a derivation of Dvoretzky's theorem and will follow two converging roads to show the counterintuitive results of measure concentration due to very high dimensionality. The theorem is derived first in a conventional mathematical fashion [16] [17], then we do the same using the Bohm model of the wave-particle duality of E-infinity theory [14].

\subsection{Conventional Derivation of Dvoretzky's Theorem}

The moral which we can learn, in fact relearn from this theorem is a well known wisdom from many counterintuitive results of geometry in higher dimensions, namely that we should in general never generalize an obvious conclusion from a low dimensional space to a higher one [16]. For instance on a flat two dimensional space any two lines will intersect in a point unless they are parallel. However the spectacular failure of this simple obvious result in three dimensional space is embarrassingly clear. Now let us start with a Euclidean ball [16]

$$
B^{n}=\left\{x \in R^{n}: \sum_{1}^{n} x_{i}^{2} \leq 1\right\} .
$$

Working in the usual way to find the volume of this $n$ dimensional ball we arrive via gamma function and Stirling formula to [16] [17]

$$
V_{n} \simeq\left(\sqrt{\frac{2 \pi e}{n}}\right)^{n} .
$$

That means for $V=1$ the radius is a very large one equal approximately to

$$
\sqrt{\frac{n}{2 \pi e}} .
$$

Now we proceed to the distribution of the mass, i.e. how the "volume" of this ball is distributed. To do that 
we estimate first the $(n-1)$ volume of a slice through the center of the unit ball. Since the radius of the ball [16] [17] is

$$
r=V_{n}^{(1 / n)}
$$

then the volume of the slice $(n-1)$ dimensional ball is given by [16] [17]

$$
\left(V_{n-1}\right)\left(r^{n-1}\right)=V_{n-1}\left(\frac{n}{V_{n}}\right)^{(n-1) / n} .
$$

Using the Stirling formula again we find that the slice has the volume $\sqrt{e}$ for very large $n$. The next question is what is the $(n-1)$ dimensional volume of a parallel slice? The slice at distance $x$ from the center is an $(n-1)$ dimensional ball with radius $\sqrt{r^{2}-x^{2}}$ so that the volume of the smaller slice is given approximately by [16] [17]

$$
V_{n-1}(\text { smaller slice })=\sqrt{e}\left(\frac{1-x^{2}}{r^{2}}\right)^{(n-1) / 2} .
$$

Since $r$ is approximately [16] [17]

$$
r \simeq \sqrt{n /(2 \pi e)}
$$

one finds [16] [17]

$$
V_{n-1}(\text { smaller slice }) \cong(\sqrt{e}) \exp \left(-\pi e x^{2}\right) .
$$

That means we obtain "mass" distribution that is almost Gaussian, with variance which surprisingly does not depend upon $n$ :

$$
\operatorname{var} \simeq 1 /(2 \pi e) .
$$

That way we conclude the following remarkable result, namely that almost all the "volume" stays within a flab of fixed width and our result announced in the introduction of the present paper follows that about $96 \%$ of the "mass", i.e. the volume lies in the slab [16] [17]

$$
\left\{x \in R^{n}: \frac{1}{2} \leq x_{1} \leq \frac{1}{2}\right\} .
$$

That means $96 \%$ is concentrated near the subspace $(n-1)$ which may be regarded as the hyper surface of an n dimensional black hole. This is a clear failure of our low dimensional intuition to anticipate what happens in high dimensional cases [16] [17].

\subsection{Derivation of Dvoretzky's Theorem from the E-Infinity Particle-Wave Duality and De Broglie-Bohm Pilot Wave Model}

The second derivation of our theorem is more or less based on physics.

In E-infinity theory the pre-quantum particle as well as the pre-quantum wave follows from the fundamental equation fixing the invariants of the noncommutative E-infinity spacetime [15]

$$
D=a+b \phi
$$

where $a, b \in Z$ and $\phi=(\sqrt{5}-1) / 2$. Setting $a=b=0$ one finds the absolutely empty set $D=0$. By contrast for $a=0$ and $b=1$ one finds the zero set $D(O)=\phi$ which models the particle while its cobordism, i.e. the surface is nothing but the empty set [12]-[15]

$$
D(-1)=1-\phi=\phi^{2}
$$

which models the quantum wave. Transferring this result to Kaluza-Klein "quantum" spacetime we note that the "inner" volume must be correlated, i.e. intersectional which is appropriate for a volume and leads to [12]-[15] 


$$
V(O)=(\phi)^{5}
$$

where $D($ Kaluza-Klein $)=5$. The outer surface, i.e. the quantum wave on the other hand is additive and noncorrelated so that the union operation is what leads to the volume [12]-[15]

$$
V(D)=5 \phi^{2} \text {. }
$$

In the above we tacitly assumed the validity of the Bohm picture of a quantum particle surrounded by a guiding quantum wave. A typical volume representative for both would be clearly the arithmetic mean

$$
\frac{\phi^{5}+5 \phi^{2}}{2}=1
$$

In turn looking at the above as energy density we see that

$$
V(O)=5 \phi^{2}=E(O)
$$

for $m=c=1$ while

$$
V(D)=5 \phi^{2} / 2=E(D)
$$

In other words $E(O)$ is our familiar ordinary measurable energy density of the quantum particle [12]-[17]

$$
E(O)=\left(\phi^{5} / 2\right) m c^{2} \cong m c^{2} / 22
$$

while $E(D)$ is our dark energy density of the quantum wave which we cannot measure [12]-[17]

$$
E(D)=\left(5 \phi^{2} / 2\right) m c^{2} \simeq m c^{2}(21 / 22) .
$$

Adding both together we obtain the celebrated result [12]-[17]

$$
E(\text { Einstein })=E(O)+E(D)=m c^{2} .
$$

Now remembering that energy and information are directly related via entropy, the preceding result is confirmation of what we obtained earlier on using Dvoretzky's theorem, namely that $96 \%$ of the information is drawn to the surface higher dimensionality rather than "diluted" by it. Needless to say, the preceding results remain valid for a rotating Kerr black hole [18].

\section{Discussion and Conclusions}

The present work is in the first place a vivid demonstration of the power of pure mathematics, in the present case the power of Dvoretzky's theorem of measure concentration, in solving problems in physics and cosmology. The mathematical literature abounds with examples demonstrating the failure of our low dimensional intuition to extrapolate from low dimensional results to higher dimensional ones. [16] and we indicated this in a 1997 paper relating the critical paradoxical dimension $D=9$ to superstring $D=10$ and sphere packing [19]. The holographic boundary and the horizon of a higher dimensional black hole is no exception. Rather than diluting the density of information, a higher dimensional black hole surface has a higher information density than a lower dimensional one. The second result reported here may be even able to dig deep into the quantum roots of Einstein's $E=m c^{2}$ and explain that way the measure ordinary energy density of the cosmos $E(O) \simeq m c^{2} / 22$ and the corresponding energy density of the so called missing dark energy $E(D) \simeq m c^{2}(21 / 22)$ [20]. Having reached this point one is justified in asking how come a non-quantum special relativity formula such as $E=m c^{2}$ could account for a quantum relativity formula $E(O)=m c^{2} / 22$ and quantum cosmology formula

$E(D)=m c^{2}(21 / 22)$. The surprizing answer is classical relativity. Indeed it seems some simple and basic classical notions such as scaling, self reference and the trivial facts that general relativity is valid for all that is relatively large compared to us humans and our laboratory equipment and similarly that quantum mechanics is valid for all what is relatively extremely small compared to us is what links Newtonian physics with relativity and quantum mechanics. This should come as a needed boost and a relatively unexpected victory for classical common sense. 


\section{References}

[1] Frolov, V.P. and Zelnikov, A. (2011) Introduction to Black Hole Physics. Oxford University Press, Oxford. http://dx.doi.org/10.1093/acprof:oso/9780199692293.001.0001

[2] Bardeen, J.M., Carter, B. and Hawking, S.W. (1973) The Four Laws of Black Hole Mechanics. Communications in Mathematical Physics, 31, 161-170. http://dx.doi.org/10.1007/BF01645742

[3] Bekenstein, J.D. (1980) Black-Hole Thermodynamics. Physics Today, 33, 24-31. http://dx.doi.org/10.1063/1.2913906

[4] Meisner, C.W., Thorne, K.S. and Wheeler, J.A. (1973) Gravitation. W.H. Freeman \& Company, San Francisco.

[5] Weinberg, S. (2008) Cosmology. Oxford University Press, Oxford.

[6] Susskind, L. and Lindesay, J. (2005) Black Holes, Information and the String Theory Revolution (The Holographic Universe). World Scientific, New Jersey.

[7] Susskind, L. (2008) The Black Hole War. Back Bay Books, New York.

[8] Horowitz, G.T., Ed. (2012) Black Holes in Higher Dimensions. Cambridge University Press, Cambridge, UK. http://dx.doi.org/10.1017/CBO9781139004176

[9] Wheeler, A. (1990) Information, Physics, Quantum: The Search for Links. In: Zurek, W., Ed., Complexity Entropy and the Physics of Information, Addison-Wesley, New York, 3-18.

[10] 'tHooft, G. (2015) G. 'tHooft Asks a Question about General Relativity on ResearchGate, Questions and Answers. October.

https://www.researchgate.net/post/In_GR_can_we_always_choose_the_local_speed_of_light_to_be_everywhere_small er_that the_coordinate_speed_of_light_Can_this_be_used_in_a_theory

[11] El Naschie, M.S. (2006) Fractal Black Holes and Information. Chaos, Solitons \& Fractals, 29, 23-35. http://dx.doi.org/10.1016/j.chaos.2005.11.079

[12] El Naschie, M.S. (2015) If Quantum "Wave” of the Universe Then Quantum "Particle” of the Universe: A Resolution of the Dark Energy Question and the Black Hole Information Paradox. International Journal of Astronomy \& Astrophysics, 5, 243-247. http://dx.doi.org/10.4236/ijaa.2015.54027

[13] El Naschie, M.S. (2015) A Resolution of the Black Hole Information Paradox via Transfinite Set Theory. World Journal of Condensed Matter Physics, 5, 249-260. http://dx.doi.org/10.4236/wjcmp.2015.54026

[14] El Naschie, M.S. (2004) A Review of E-Infinity and the Mass Spectrum of High Energy Particle Physics. Chaos, Solitons \& Fractals, 19, 209-236. http://dx.doi.org/10.1016/S0960-0779(03)00278-9

[15] Connes, A. (1994) Noncommutative Geometry. Academic Press, San Diego.

[16] Levy, S., Ed. (1997) Flavors of Geometry. Cambridge University Press, Cambridge, UK.

[17] El Naschie, M.S. (2015) Banach Spacetime-Like Dvoretzky Volume Concentration as Cosmic Holographic Dark Energy. International Journal of High Energy Physics, 2, 13-21. http://dx.doi.org/10.11648/j.ijhep.20150201.12

[18] El Naschie, M.S. (2015) Kerr Black Hole Geometry Leading to Dark Matter and Dark Energy via E-Infinity Theory and the Possibility of Nano Spacetime Singularity Reactor. Natural Science, 7, 210-225. http://dx.doi.org/10.4236/ns.2015.74024

[19] El Naschie, M.S. (1997) Remarks on Super Strings, Fractal Gravity, Nagasawa’s Diffusion and Cantorian Spacetime. Chaos, Solitons \& Fractals, 8, 1873-1886.

[20] El Naschie, M.S. (2014) Why E Is Not Equal $m c^{2}$. Journal of Modern Physics, 5, 743-750. http://dx.doi.org/10.4236/jmp.2014.59084 